Int. J. Odontostomat., 10(1):49-53, 2016.

\title{
Osteomielitis en el Cóndilo Mandibular y su Relación con la Anemia
}

\author{
Osteomyelitis in the Condyle of the Jaw and its Relationship with Anemia
}

\author{
Claudia Letelier F."; Günther Preisler E.."*; Paulo García C..", \& Fernando Salinas R.
}

LETELIER, F. C.; PREISLER, E. G.; GARCÍA, C. P. \& SALINAS, R. F. Osteomielitis en el cóndilo mandibular y su relación con la anemia. Int. J. Odontostomat., 10(1):49-53, 2016.

RESUMEN: La osteomielitis se define como una inflamación progresiva del hueso, que se produce con mayor frecuencia en los huesos largos del cuerpo. Su etiología se relaciona con factores locales y/o sistémicos los que incluyen la anemia. La osteomielitis resultante de la anemia de células falciformes genera que la sangre sea más viscosa produciendo un bajo flujo sanguíneo conduciendo a infarto isquémico y necrosis del hueso, rara vez causado en los huesos faciales. Paciente ingresado a urgencia por un aumento de volumen en la región preauricular izquierda de un mes de evolución, blando, fluctuante, límites mal definidos, de $5 \times 4 \mathrm{~cm}$ de diámetro, afebril, sin cambios de coloración. Presentaba trismus y dolor a la palpación. Hace cinco meses había sido tratado por una osteomielitis frontoparietal izquierda con absceso subgaleal. Antecedentes mórbidos del paciente: alcohólico crónico y anemia en tratamiento. El paciente fue hospitalizado con tratamiento de Clocaxiclina endovenoso, coleccionándose en la región preauricular. Se realizó la biopsia dando como resultado osteomielitis de cóndilo mandibular. La osteomielitis presentada en el cóndilo mandibular pudo ser recidiva de la osteomielitis anterior o haber sido causada por la anemia. Sin embargo, la osteomielitis frontoparietal se presentó sin causa aparente, pudiendo relacionarla con la anemia que cursaba.

PALABRAS CLAVE: osteomielitis, cóndilo mandibular, anemia.

\section{INTRODUCCIÓN}

Osteomielitis (OM) se define como una inflamación progresiva del hueso (Baur et al., 2015; Baltensperger \& Eyrich, 2008), que se produce con mayor frecuencia en los huesos largos del cuerpo (Baltensperger \& Eyrich; Kellher et al., 1996; Adeyemo et al., 2011). Su etiología se relaciona con factores locales, como el trauma, propagación de infecciones a distancia, cirugías y la infección dental. Dentro de los factores sistémicos que predisponen a los individuos a OM incluyen la diabetes, anemia, desnutrición, enfermedades malignas (Baur et al.; Baltensperger \& Eyrich; Kellher et al.; Adeyemo et al.).

La osteomielitis resultante de la anemia de células falciformes ha sido reconocida desde 1925 (Baltensperger \& Eyrich), donde las células deformes generan que la sangre sea más viscosa produciendo un bajo flujo sanguíneo y una estasis crónica de sangre en los capilares (Kellher et al.; Adeyemo et al.;
Neville et al., 2008). El estasis significativo y prolongado conduce a infarto isquémico y necrosis del hueso. La osteomielitis como resultado de la enfermedad de células falciformes rara vez afecta a los huesos faciales. De éstos, la mandíbula es la más afectada (Baltensperger \& Eyrich, Kellher et al.; Adeyemo et al.).

El objetivo de nuestro reposrte es presentar un caso de un paciente con anemia que presentó osteomielitis de cóndilo mandibular sin asociación a trauma o infecciones dentarias.

\section{PACIENTE Y MÉTODO}

Hombre 60 años de edad, paramédico, fue ingresado al servicio de urgencias por asimetría facial con un aumento de volumen en la región preauricular

* Cirujano Dentista. Docente Escuela de Odontología, Universidad Austral de Chile, Valdivia, Chile.

"Especialista en Cirugía Maxilofacial, Hospital Base Valdivia, Valdivia, Chile.

Fuente de apoyo financiero: El estudio fue realizado con el financiamiento de los autores y la ayuda del Hospital base Valdivia, Chile. 
izquierda de un mes de evolución. La inflamación era blando, fluctuante, con límites mal definidos, de aproximadamente $5 \times 4$ $\mathrm{cm}$ de diámetro, afebril, sin cambios de coloración de la piel. Se acompañaba de trismus, dolor a la palpación y sin alteraciones neurológicas (Fig. 1).

Dentro de su historia de evolución, el paciente hace 5 meses ingresó al servicio de urgencia por un aumento de volumen frontoparietal izquierdo de consistencia blanda sin causa aparente. La tomografía computadorizada (TC) mostró un tumor frontoparietal derecho con calcificación y edema periolesional (Fig. 2). Además, se vio un aumento de volumen subgaleal frontoparietal izquierdo y defecto óseo en el centro. Al día siguiente del ingreso de urgencia drenó espontáneamente el contenido purulento donde se tomó cultivo y biopsia del tejido galeal y hueso. Se realizó aseo de la zona instalando un drenaje y se inició tratamiento con Cloxaciclina endovenoso por dos semanas. Su evolución fue buena, se retiró el drenaje a los 11 días postquirúrgico. El diagnóstico de egreso fue osteomielitis frontoparietal izquierdo con absceso subgaleal.

Dentro de los antecedentes mórbidos el paciente era alcohólico crónico y con antecedentes de anemia en tratamiento. Sin antecedentes quirúrgicos previos aparte de la osteomielitis frontoparietal.

Luego de tomar conocimiento del caso el paciente fue hospitalizado con tratamiento de Clocaxiclina endovenoso, siguiendo la misma línea de tratamiento antes utilizada. En la TC se observó en la articulación temporomandibular (ATM) izquierda elementos erosivos del cóndilo mandibular con pérdida de la cortical y se aprecia aumento de volumen de captación de contraste a nivel de partes blandas periféricas en relación a la misma zona (Fig. 3).

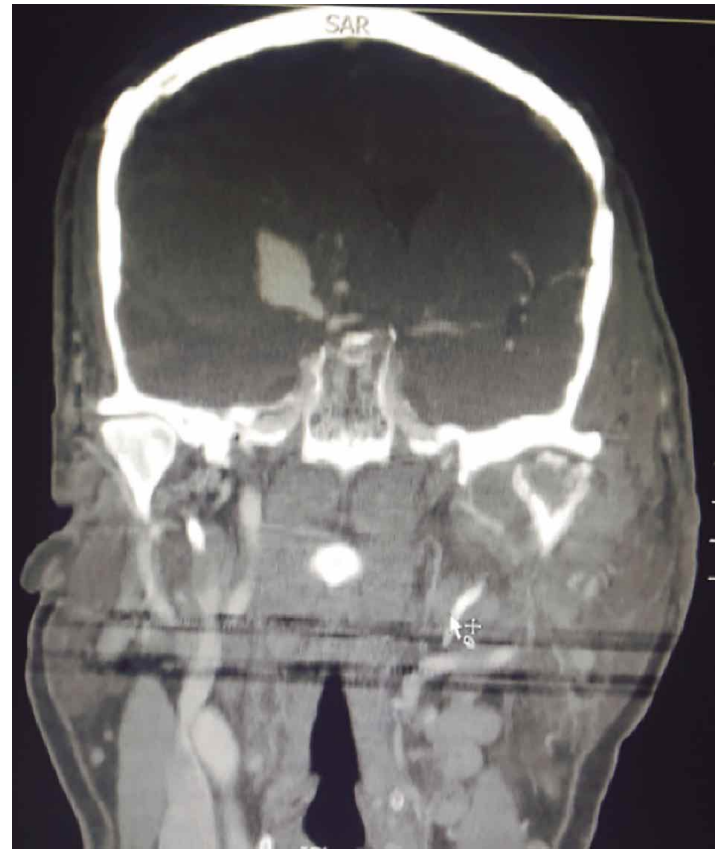

Fig. 3. Elementos erosivos del cóndilo mandibular izquierdo con pérdida de la cortical y aumento de volumen de partes blandas periféricas.

Tres días después del tratamiento antibiótico hubo una disminución importante del aumento de volumen, coleccionándose en la región preauricular, con límites definidos, no fluctuante, de consistencia dura. Se realizó la biopsia bajo anestesia general con un abordaje preauricular izquierdo (Fig. 4).

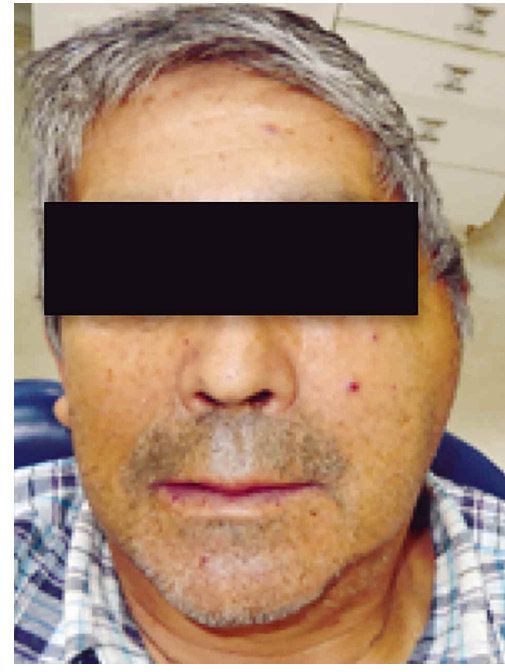

Fig. 1. Aumento de volumen región preauricular izquierda.

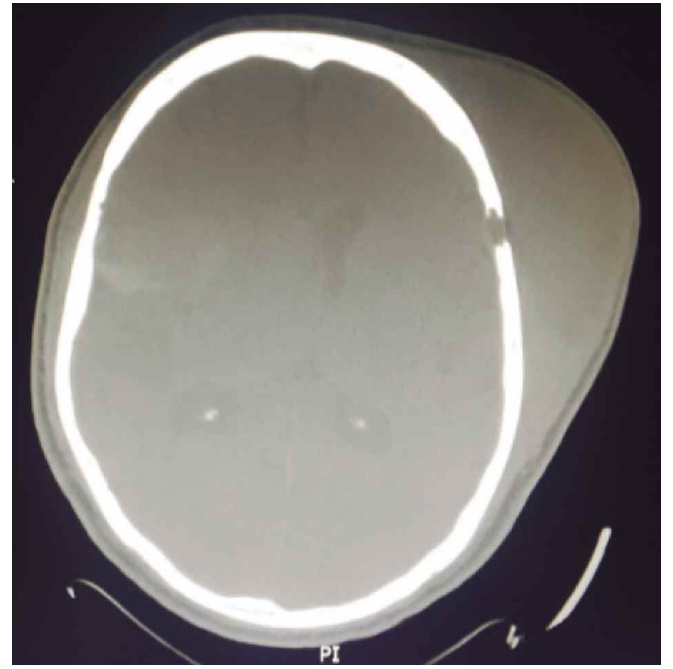

Fig. 2. Aumento de volumen subgaleal frontoparietal izquierdo. 


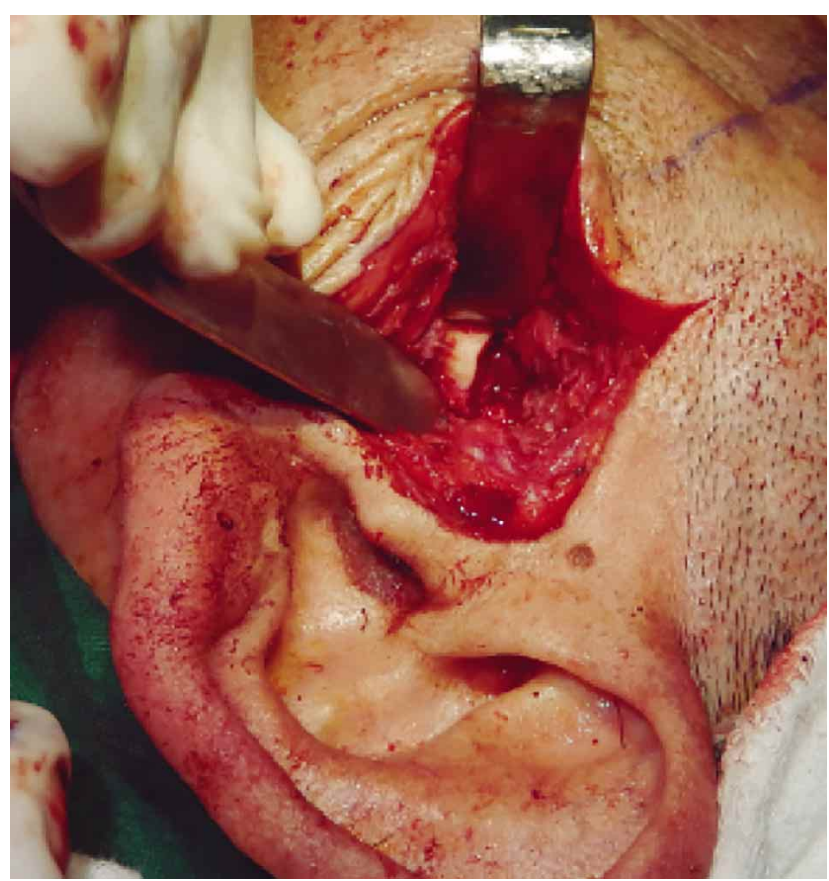

Fig. 4. Extirpación de cóndilo mandibular izquierdo.

En el control al día siguiente se observó buena dinámica mandibular lateralidad y ausencia de alteraciones neurológicas. Se indicó ejercicios de apertura y lateralidad mandibular y se continuó con controles por tres meses. El resultado de la biopsia fue osteomielitis de cóndilo mandibular.

\section{DISCUSIÓN}

La OM se define como la inflamación progresiva del hueso y la médula ósea, esta se encuentra más comúnmente en los huesos largos y con poca frecuencia en los huesos de la cara (Baur et al.; Baltensperger \& Eyrich; Kellher et al.; Adeyemo et al.). Se clasifica como aguda, subaguda y crónica según su presentación clínica y dependiendo de esta se define su tratamiento (Baltensperger et al., 2004; Zorzan et al., 2011). La OM aguda evoluciona a lo largo de días o en pocas semanas, y se manifiesta por un inicio repentino de los síntomas sistémicos, que incluyen fiebre, leucocitosis, linfadenopatía e hinchazón del área. Si la fase aguda pasa sin el cese completo de los síntomas puede aparecer las formas subaguda o crónicas de la enfermedad (Zorzan et al.; Petrikowski et al., 1995). La OM subaguda se desarrolla dentro de semanas y es una infección crónica de bajo grado del hueso que se caracteriza por la falta de manifestacio- nes sistémicas y un inicio insidioso (Petrikowski et al.). Las formas agudas y subagudas de la enfermedad por lo general responden bien a una línea de tratamiento antibiótico (Zorzan et al.; Petrikowski et al.). La OM crónica es una infección recurrente y persistente que evoluciona a lo largo de meses o años y se caracteriza por la inflamación de bajo grado, la presencia de secuestros óseos, nueva oposición ósea, y trayecto fistuloso (Petrikowski et al.). Esta forma requiere la intervención quirúrgica con tratamiento antibiótico de amplio espectro a largo plazo (Baltensperger et al.; Petrikowski et al.).

En el caso presentado previamente se hizo la hipótesis diagnostica como OM crónica por su evolución clínica y radiográfica, además de su diagnóstico previo de OM parietal que luego se confirmó con la biopsia.

La TC se ha utilizado cada vez más para evaluar la presencia y extensión de OM mandibular incluyendo la inflamación de tejidos blandos. Los hallazgos de la TC en la fase aguda de la OM consisten en un área de osteólisis que en un principio se limita a hueso esponjoso mientras que en el hueso cortical puede verse afectada como resultado de desmineralización y erosión local. Generalmente entre la primera y segunda semana de evolución de la OM se detectan hallazgos como alteraciones en el trabeculado óseo, desmineralización, erosión y adelgazamiento de las corticales acompañados con abscesos (Baltensperger \& Eyrich). Cuando continúa hasta la cuarta semana nos podemos encontrar con secuestros óseos y zonas esclerosadas junto con fracturas como complicaciones asociadas (Baltensperger \& Eyrich).

La OM mandibular es una rara complicación de individuos que padecen anemia, la cual ha sido poco reportada y estudiada en los huesos faciales (Baur et al.; Baltensperger \& Eyrich; Kellher et al.; Adeyemo et al.). La ubicación más común dentro de los huesos faciales es en los huesos que tienen relación a los dientes y principalmente en la mandíbula por ser más avascular que el maxilar (Baur et al.; Baltensperger \& Eyrich; Kellher et al.; Neville et al.). Sin embargo, en este caso se presentó en el cóndilo mandibular. La mayoría de los autores que relaciona la anemia con la osteomielitis coinciden con que la etiología se genera debido a la anemia hemolítica crónica, a la vasooclusión y al aumento de viscosidad de la sangre que va a dar como resultado el bajo flujo sanguíneo y una estasis crónico de sangre lo que conduce a infar- 
to isquémico y necrosis del hueso (Baur et al.; Baltensperger \& Eyrich; Kellher et al.; Neville et al.). Éste tejido óseo necrótico a su vez crea un ambiente favorable para el crecimiento de bacterias de la región oral.

En la osteomielitis de los huesos faciales, es causada por microorganismos siendo el Streptococcus spp. el más común, seguido por Actinomyces, Bacteroides y Lactobacillus (Baur et al.; Baltensperger \& Eyrich). Es por esto que debe realizarse una terapia antibiótica antes de lacirugía para obtener una caracterización bacteriana más precisa y garantizar un resultado de tratamiento más favorable. Está establecido en la literatura que se requiere una terapia antibiótica a largo plazo para el tratamiento de la osteomielitis, sin embargo, la duración exacta sigue siendo controversial. Según Rao et al. (2011) cuatro a seis semanas de terapia con antibióticos después de la cirugía es suficiente, mientras que Kim \& Jang (2001) presentaron resultados favorables con la aplicación de antibióticos durante ocho semanas después de la cirugía. Otros autores recalcan que es más beneficioso para determinar la duración del tratamiento antibiótico el cumplimiento del paciente y la evolución clínica y sistémica, basada en los marcadores de inflamación, como la proteína $\mathrm{C}$ reactiva (Baur et al.).

El tratamiento antibiótico eficaz es un componente indispensable para el tratamiento de la osteomielitis que asegura un mejor resultado del tratamiento; sin embargo, no tiene ningún efecto sobre el hueso necrótico, por lo tanto, independientemente de la causa siempre debe incluir la extirpación quirúrgica como parte del tratamiento del hueso necrótico en la mandíbula (Baur et al.; Baltensperger \& Eyrich; Neville et al.).

Dentro de las limitaciones de este caso existe la duda de si la osteomielitis de cóndilo mandibular se relaciona con la osteomielitis frontoparietal presentada meses antes, o que ambas osteomielitis fueron causadas de forma independiente por la anemia de base del paciente. Sin embargo, la osteomielitis frontoparietal no presenta factores etiológicos locales por lo que se podría establecer que sí fue causada por la anemia. Otra limitación incluye el corto seguimiento del paciente luego de la cirugía.

Como conclusión, es importante tener en conocimiento esta relación para establecer un diagnóstico correcto y por ende un tratamiento oportuno, con el uso de antibióticos y tratamiento quirúrgico. Además, hay que tener en consideración que esta enfermedad y su relación con la anemia puede afectar a todos los huesos, incluso los huesos faciales como el cóndilo mandibular.

\section{AGRADECIMIENTOS}

Nuestros sinceros agradecimientos al hospital base Valdivia y a sus funcionarios que facilitaron los exámenes y la realización del caso clínico.

LETELIER, F. C.; PREISLER, E. G.; GARCÍA, C. P. \& SALINAS, R. F. Osteomyelitis in the condyle of the jaw and the relationship with anemia. Int. J. Odontostomat., 10(1):4953, 2016.

ABSTRACT: Osteomyelitis is defined as a progressive inflammation of the bone, which occurs most frequently in the long bones of the body. Its etiology is related to local and / or systemic factors that include anemia. Osteomyelitis resulting from sickle cell anemia results in the blood being more viscous producing a low blood flow leading to ischemic attack and bone necrosis. Patient presented with increased volume in the left preauricular region following one month of evolution, soft, fluctuating, ill-defined limits, $5 \times 4 \mathrm{~cm}$ in diameter, afebrile, without discoloration. He had trismus, tenderness and had been admitted to the emergency room five months earlier, for osteomyelitis with subgaleal abscess. Patient's background included anemia and treatment for chronic alcoholism. The patient was hospitalized with intravenous treatment of cloxacilline, resulting in increased preauricular volume with defined limits. Results following a biopsy showed condyle osteomyelitis. Osteomyelitis presented in the condyle may be the recurrence of osteomyelitis or may have been caused by the anemia. However, frontoparietal osteomyelitis has no apparent cause and could be associated with anemia. anemia.

KEY WORDS: osteomyelitis, mandibular condyle,

\section{REFERENCIAS BIBLIOGRÁFICAS}

Adeyemo, T. A.; Adeyemo, W. L.; Adediran, A.; Akinbami, A. J. \& Akanmu, A. S. Orofacial manifestations of hematological disorders: anemia and hemostatic disorders. Indian J. Dent. Res., 22(3):454-61, 2011.

Baltensperger, M.; Grätz, K.; Bruder, E.; Lebeda, R.; Makek, M. \& Eyrich, G. Is primary chronic osteomyelitis a uniform disease? Proposal of a classification based on a retrospective analysis of patients treated in the past 30 years. J. Craniomaxillofac. Surg., 32(1):43-50, 2004. 
Baltensperger, M. M. \& Eyrich, G. K. (Eds.). Osteomyelitis of the Jaws. Berlin, Springer, 2009.

Baur, D. A.; Altay, M. A.; Flores-Hidalgo, A.; Ort, Y. \& Quereshy, F. A. Chronic osteomyelitis of the mandible: diagnosis and management--an institution's experience over 7 years. J. Oral Maxillofac. Surg., 73(4):655-65, 2015.

Kellher, M.; Bishop, K. \& Briggs, P. Oral complications associated with sickle cell anemia: a review and case report. Oral Surg. Oral Med. Oral Pathol. Oral Radiol. Endod., 82(2):225-8, 1996.

Kim, S. G. \& Jang, H. S. Treatment of chronic osteomyelitis in Korea. Oral Surg. Oral Med. Oral Pathol. Oral Radiol. Endod., 92(4):394-8, 2001.

Neville, B. W.; Damm, D. D.; Allen, C. \& Bouquot, J. Oral and Maxillofacial Pathology. $3^{a}$ ed. St. Louis, Saunders Elsevier, 2008.

Petrikowski, C. G.; Pharoah, M. J.; Lee, L. \& Grace, M. G. Radiographic differentiation of osteogenic sarcoma, osteomyelitis, and fibrous dysplasia of the jaws. Oral Surg. Oral Med. Oral Pathol. Oral Radiol. Endod., 80(6):744-50, 1995

Rao, N.; Ziran, B. H. \& Lipsky, B. A. Treating osteomyelitis: antibiotics and surgery. Plast. Reconstr. Surg., 127 Suppl. 1:177S-87S, 2011.

Zorzan, G.; Tullio, A.; Bertolini, F. \& Sesenna E. Osteosarcoma of the mandibular condyle: Case report. J. Oral Maxillofac. Surg., 59(5):574-7, 2011.
Dirección para Correspondencia: Dra. Claudia Letelier Fuentealba Cirujano Dentista Docente Escuela de Odontología Universidad Austral de Chile Rudloff 1640

Valdivia

CHILE

Email: cletelierfuentealba@gmail.com

Recibido : 25-10-2015

Aceptado: 13-01-2016 